\title{
Manifestaciones gastrointestinales de pacientes infectados con el nuevo Coronavirus SARS-CoV-2
}

\author{
Luis Antonio Díaz P. ${ }^{l}$ y Alberto Espino E. ${ }^{1}$
}

\section{Gastrointestinal manifestations in infected patients with the novel Coronavirus SARS-CoV-2}

The pandemic of the novel coronavirus SARS-CoV-2 has caused more than 2.5 millions of confirmed cases worldwide, with a mortality rate around 7\%. Most cases appear to be mild with the most common symptoms being fever, dry cough, myalgia, fatigue, and dyspnea but can result in more severe disease. Also, there are described gastrointestinal manifestations: anorexia $27 \%$, diarrhea $12 \%$, nausea/vomiting $10 \%$, and abdominal pain $9 \%$. In Chile, diarrhea has been described in 7.3\%, and abdominal pain 3.7\%. The primary mechanism proposed is the entry of SARS-CoV-2 into the enterocytes binding the Angiotensin-converting enzyme 2 (ACE2), which is widely distributed among the gastrointestinal tract. Also, SARS-CoV-2 can cause elevated liver enzymes in up to $40 \%$ of cases. This alteration could also be caused by the binding to the ACE2 receptor in hepatocytes and cholangiocytes. Gastrointestinal symptoms and elevated liver enzymes are more frequent in severe cases. Viral RNA has been detected in stools, which may suggest a possible fecal-oral transmission. It is essential to pay attention to gastrointestinal manifestations because they are frequent and more prevalent in severe cases. The presence of viral RNA in stool forces us to take preventive measures faced with a possible mechanism of fecal-oral transmission, mainly in the gastroenterology and endoscopy services.

Key words: SARS-CoV-2, coronavirus COVID-19, gastrointestinal.

\section{Resumen}

La pandemia por el nuevo coronavirus SARS-CoV-2 ha causado más de 2,5 millones de casos confirmados en el mundo, con una mortalidad cercana al 7\%. La mayoría de los casos parecen ser leves y los síntomas más comunes son fiebre, tos seca, mialgia, fatiga y disnea, pero pueden provocar una enfermedad más grave. También se han descrito síntomas gastrointestinales: anorexia $27 \%$, diarrea 12\%, náuseas/vómitos 10\%, y dolor abdominal 9\%. En Chile, se ha descrito diarrea en 7,3\%, y dolor abdominal 3,7\%. El principal mecanismo fisiopatológico propuesto es el ingreso al enterocito utilizando la Enzima Convertidora de Angiotensina 2 (ECA2) como receptor, el cual es ampliamente distribuido a lo largo del tracto gastrointestinal. También se ha descrito alteración de pruebas hepáticas hasta en $40 \%$ de los casos. Esta alteración también podría ser causada por el ingreso a los hepatocitos y colangiocitos mediante la ECA2. Los síntomas gastrointestinales, y las alteraciones de pruebas hepáticas son más frecuentes en los pacientes graves. Se ha detectado la presencia de ARN viral en deposiciones de pacientes, lo que podría sugerir una posible vía de transmisión fecal-oral. Es importante prestar atención a las manifestaciones gastrointestinales, ya que son comunes y más frecuentes en pacientes graves. La presencia viral en las heces nos obliga a tomar medidas de precaución y prevención de un posible mecanismo de transmisión fecal-oral, especialmente en los servicios de gastroenterología y endoscopia.

Palabras clave: SARS-CoV-2, coronavirus COVID-19, gastrointestinal.
${ }^{1}$ Departamento de Gastroenterología, Escuela de Medicina, Pontificia Universidad Católica de Chile. Santiago, Chile.

Recibido:

Aceptado:

Correspondencia a: Dr. Alberto Espino Profesor Asistente Departamento de Gastroenterología Pontificia Universidad Católica de Chile Jefe Unidad Procedimientos Digestivos. Red Salud UC-Christus

Diagonal Paraguay 362. 4to piso. Santiago, Chile. aoespino@uc.cl 


\section{Introducción}

Los coronavirus son una gran familia de virus de ARN con la capacidad de infectar humanos y animales. Desde su descripción por primera vez en el año 1966, se han identificado cuatro tipos de coronavirus que causan habitualmente infecciones respiratorias altas $^{1,2}$. En los últimos años, se han identificado otros dos miembros de esta familia que con mayor frecuencia causan insuficiencia respiratoria aguda: Severe Acute Respiratory Syndrome-CoV (SARS-CoV) el 2002 y Middle East Respiratory Syndrome-CoV (MERS-CoV) el 2012. En relación con el SARS-CoV, es importante destacar que alcanzó una mortalidad de $10,5 \%$ en 8098 pacientes $^{3}$. Actualmente, el nuevo coronavirus SARS-CoV-2 es el causal de la pandemia COVID-19 (por sus siglas en inglés Coronavirus Disease 2019), cuyo primer caso fue originado en Wuhan (China) durante diciembre 2019. A la fecha, se han descrito más de 2.592 .857 casos confirmados y 181.083 muertes a nivel mundial (mortalidad cercana al 7\%) ${ }^{4}$. En Chile, se han confirmado más de 11.296 casos, lamentando la muerte de 160 personas (mortalidad cercana al 1,4\%) ${ }^{5}$. El virus SARS-CoV-2 puede presentar predominantemente síntomas respiratorios y constitucionales ${ }^{6-8}$. Sin embargo, también se han descrito manifestaciones gastrointestinales, el cual es el objetivo de esta revisión.

\section{Manifestaciones clínicas generales y gastrointestinales}

La presentación clínica de COVID-19 puede variar desde pacientes asintomáticos, con síntomas respiratorios leves inespecíficos, a una disfunción de órganos grave, como el síndrome de dificultad respiratoria aguda (SDRA) que puede conducir a la muerte. El período de incubación del SARS-CoV-2 promedio es de 5,2 días, pero puede variar de 2 a 14 días. Los síntomas más comunes son fiebre (83-98\%), tos (46-82\%), mialgia/fatiga (11-44\%) y disnea (31\%). La mayoría de los casos confirmados han sido clasificados según gravedad en leves o moderados $(80+\%)$, graves $14 \%$ y críticos $5 \%$. Dentro de los factores de riesgo para desarrollar enfermedad grave que requiere de hospitalización se han descrito, edad mayor $\geq 65$ años, tener comorbilidades crónicas como hipertensión, diabetes, obesidad, tabaquismo, enfermedad pulmonar y enfermedad cardiovascular ${ }^{1,7-9}$.

Los pacientes además pueden presentar manifestaciones gastrointestinales cuya frecuencia varían según la población estudiada y la gravedad del cuadro $^{10,11}$. En Chile, un informe del MINSAL al 11 de abril 2020, mostró que el $11 \%$ de los casos de SARS-CoV-2 confirmados presentaron algún síntoma gastrointestinal: un $7,3 \%$ presentó diarrea y un 3,7\% dolor abdominal ${ }^{5}$. Por otro lado, un meta-análisis de Cheung KS et al., que incluyó 4.243 pacientes de población predominantemente asiática y europea, evidenció una prevalencia de manifestaciones gastrointestinales del 17,6\% (IC 95\%, 12,3-24,5\%) ${ }^{12}$. El síntoma más frecuente fue la anorexia con $27 \%$, seguido de diarrea $12 \%$, náuseas/vómitos $10 \%$, y dolor abdominal 9\%. La prevalencia de los síntomas gastrointestinales fue mayor en pacientes con enfermedad grave que en pacientes sin gravedad $(17 \%$ versus $12 \%$, respectivamente). Estas frecuencias fueron similares en población adulta, pediátrica y mujeres embarazadas ${ }^{13,14}$.

En relación a la diarrea en pacientes infectados con SARS-CoV-2, esta puede fluctuar entre 1 a 14 días, con una duración promedio de 5,4 días y una frecuencia de 4,3 veces al día ${ }^{15}$. No obstante, en aquellos pacientes con SARS-CoV-2 que presenten diarrea, siempre debemos descartar que el origen de la diarrea sea por el uso de fármacos u otras comorbilidades, pues varios de los fármacos usados para el tratamiento de esta infección (arbidol, fosfato de cloroquina, lopinavir y remdesivir, e incluso la medicina tradicional china) pueden causar diarrea como reacción adversa ${ }^{12,16}$.

El dolor abdominal y la anorexia son síntomas relevantes, ya que fueron más frecuentes en aquellos pacientes que requirieron cuidados intensivos ${ }^{7}$. Además, en un trabajo publicado por Jin X. et al., se demostró que aquellos pacientes que tuvieron síntomas gastrointestinales, presentaron tasas significativamente más altas de fiebre $>38,5^{\circ} \mathrm{C}$, fatiga, disnea y cefalea ${ }^{11}$. Un estudio mostró que los pacientes con síntomas digestivos han presentado un tiempo significativamente mayor desde el inicio hasta el ingreso al hospital que pacientes sin síntomas digestivos (9,0 días versus 7,3 días). Además, este estudio mostró que el $47 \%$ de casos infectados con SARS-CoV-2 presentaban síntomas respiratorios con síntomas digestivos; y hasta en un $3 \%$ de los casos hubo presencia de síntomas digestivos, pero sin síntomas respiratorios. Dentro de este mismo estudio se demostró que a medida que aumenta la gravedad de la enfermedad, los síntomas digestivos se hicieron más pronunciados ${ }^{17}$.

Por lo tanto, debido a las potenciales implicancias en el pronóstico de pacientes SARS-CoV-2 con manifestaciones gastrointestinales, es importante que los profesionales de la salud tengan un alto grado de sospecha en su práctica clínica diaria.

\section{Mecanismos fisiopatológicos}

Es importante destacar que gran parte de las manifestaciones gastrointestinales del SARS-CoV-2 
han sido descritas previamente en la infección por SARS-CoV, especialmente la diarrea. Sin embargo, la frecuencia de diarrea fue bastante más alta en la infección por SARS-CoV, alcanzando una frecuencia del $31,6 \%{ }^{18}$. El SARS-CoV y SARS-CoV-2 comparten aproximadamente el $78 \%$ de la identidad a nivel de sus aminoácidos ${ }^{19}$. En base a esto, se ha propuesto que el SARS-CoV ingresa a las células utilizando la Enzima Convertidora de Angiotensina 2 (ECA2) como receptor, la cual es ampliamente distribuida a lo largo del tracto respiratorio y gastrointestinal. De hecho, la ECA2 se expresa de forma intensa en los enterocitos a nivel proximal y distal, permitiendo potencialmente la entrada a través del tracto gastrointestinal ${ }^{20}$. Por ende, debido a su parcial similitud, el SARS-CoV-2 también podría ingresar a las células mediante este mecanismo ${ }^{21,22}$. Debido a que la ECA2 modula la inflamación intestinal, el SARS-CoV-2 puede causar la interrupción de la función ECA2 y provocar diarrea ${ }^{23}$. Un reciente estudio demostró la tinción intracelular de la proteína viral nucleocápside y la expresión proteica ECA2 en las células epiteliales del duodeno, estómago y recto humano ${ }^{24}$. Por lo tanto, toda la evidencia anteriormente expuesta sugiere que los receptores ECA2 podrían actuar como el punto de entrada del virus SARS-CoV-2 en el tracto intestinal. Por lo tanto, es importante potenciar la investigación relacionada SARS-CoV-2 y el tracto digestivo.

\section{Manifestaciones hepáticas}

Desde el punto de vista hepático, hasta el $40 \%$ de los pacientes puede presentar elevación de aminotransferasas (GOT, GPT) o Bilirrubina. Además, estas alteraciones son más frecuentes en pacientes críticos $(67,4 \%$ en críticos versus $34,1 \%$ no críti$\cos )^{25,26}$. El compromiso hepático del SARS-CoV-2 podría estar explicado por un daño transitorio del hepatocito. No está claro si el SARS-CoV-2 deteriora o no las células del hígado y los conductos biliares que expresan ECA2. Hay que considerar que los medicamentos de terapia antiviral que se han probado para tratar COVID-19, como lopinavir, ritonavir, ribavirina o la medicina tradicional china, pueden causar daño hepático inducido por fármacos. Asimismo, los antipiréticos y analgésicos, combinados con antibióticos utilizados para infecciones bacterianas como las quinolonas y las cefalosporinas también pueden contribuir a generar daño. Otros factores que potencialmente pueden influir en la alteración de las pruebas hepáticas son el deterioro de la circulación y la oxigenación. La mayoría de las manifestaciones hepáticas relacionadas con SARS-CoV-2 son leves $\mathrm{y}$ solo necesitan una estrecha vigilancia sin intervención ${ }^{27}$.

\section{Potencial mecanismo de transmisión fecal-oral}

Por otro lado, se ha detectado la presencia de ARN viral en deposiciones de pacientes, lo que podría sugerir una posible vía de transmisión fecaloral $^{28}$. La tasa de positividad de ARN viral concomitante de las muestras de heces y respiratorias fue del $48 \%$. En estudios que informaron pruebas en serie, el $70 \%$ de los pacientes presentaron ARN de heces persistentemente positivo después de que las pruebas respiratorias se volvieron negativas, pudiendo durar hasta $\geq 33$ días después del inicio de la enfermedad. En un estudio de Hong Kong la carga viral de ARN fue detectada en el 38,5\% y 8,7\% de los pacientes COVID-19 con y sin diarrea, respectivamente $(\mathrm{p}=0,02)^{29}$. Wölfel $\mathrm{R}$. et al. ${ }^{30}$ proporcionan un análisis virológico detallado de una cohorte de 9 casos de COVID-19 y sus contactos para determinar la replicación específica del sitio del cuerpo y la infectividad del virus durante varias semanas. La diseminación faríngea del virus fue muy alta durante la primera semana de síntomas y luego disminuyó rápidamente. El virus infeccioso se aisló fácilmente de muestras derivadas de faringe y pulmón. Las muestras de sangre y orina nunca demostraron ningún virus. Se encontró una alta concentración de ARN de SARS-CoV-2 en muestras de heces de individuos infectados, pero no se pudo recuperar ningún virus infeccioso de las heces. Este estudio pone en duda la posible vía de transmisión fecal-oral, sin embargo, la confirmación final de esta hipótesis tendrá que esperar estudios a mayor escala.

\section{Conclusiones}

En suma, los profesionales de la salud deben ser conscientes de que los síntomas gastrointestinales en pacientes con COVID-19 son comunes y que podrían ser la manifestación de presentación, pudiendo ser subvalorados. Los síntomas gastrointestinales son más frecuentes en pacientes de mayor gravedad, lo que tiene implicancias pronósticas, requiriendo una monitorización más estricta. La presencia viral en las heces ocurre durante el curso de la enfermedad y puede persistir más allá de esto, hecho importante para tomar medidas de precaución y prevención de un posible mecanismo de transmisión fecal-oral, actualmente en investigación. Los equipos de gastroenterología y endoscopia deben tener especial precaución durante la realización de endoscopias en pacientes con COVID-19 incluso durante el período posterior a la recuperación clínica, usando adecuadamente el equipo de protección personal. 


\section{Referencias}

1.- Ungaro RC, Sullivan T, Colombel J-F, Patel G, What Should Gastroenterologists and Patients Know About COVID-19?, Clinical Gastroenterology and Hepatology 2020. doi: https://doi. org/10.1016/j.cgh.2020.03.020. [Epub ahead of print]

2.- Lim YX, Ng YL, Tam JP, Liu DX. Human Coronaviruses: A Review of Virus-Host Interactions. Diseases. 2016; 4:26.

3.- Wilder-Smith A, Chiew CJ, Lee VJ. Can we contain the COVID-19 outbreak with the same measures as for SARS? Lancet Infect Dis. 2020. doi: 10.1016/ S1473-3099(20)30129-8. [Epub ahead of print]

4.- World Health Organization. Coronavirus disease (COVID-2019) situation reports. Consultado el 22 de abril 2020. Disponible en: https://www. who.int/emergencies/diseases/novelcoronavirus-2019/situation-reports

5.- Ministerio de Salud de Chile. SITUACIÓN EPIDEMIOLÓGICA COVID-19. CHILE. Disponible en: https://www.minsal.cl/nuevocoronavirus-2019-ncov/informeepidemiologico-covid-19/ (Consultado el 22 de abril 2020).

6.- Hick JL, Biddinger PD. Novel Coronavirus and Old Lessons - Preparing the Health System for the Pandemic. N Engl J Med 2020. doi: 10.1056/ NEJMp2005118. [Epub ahead of print].

7.- Wang D, Hu B, Hu C, Zhu F, Liu X, Zhang J, et al. Clinical Characteristics of 138 Hospitalized Patients With 2019 Novel Coronavirus-Infected Pneumonia in Wuhan, China. Jama 2020. doi: 10.1001/jama.2020.1585 [Epub ahead of print].

8.- Guan W-J, Ni Z-Y, Hu Y, Liang WH, Ou CQ, He JX, et al. Clinical Characteristics of Coronavirus Disease 2019 in China. N Engl J Med 2020. doi: 10.1056/ NEJMoa2002032. [Epub ahead of print].

9. Jordan RE, Adab P, Cheng KK. Covid-19: risk factors for severe disease and death. BMJ. 2020;368:m1198.

10.- Gu J, Han B, Wang J. COVID-19: Gastrointestinal manifestations and potential fecal-oral transmission. Gastroenterology 2020 doi: 10.1053/j. gastro.2020.02.054. [Epub ahead of print]
11.- Jin X, Lian JS, Hu JH, Gao J, Zheng L, Zhang YM, et al. Epidemiological, clinical and virological characteristics of 74 cases of coronavirus-infected disease 2019 (COVID-19) with gastrointestinal symptoms. Gut 2020. doi: https://doi. org/10.1136/gutjnl-2020-320926 [Epub ahead of print].

12.- Cheung KS, Hung IFN, Chan PPY, Lung KC, Tso E, Liu R, et al. Gastrointestinal Manifestations of SARS-CoV-2 Infection and Virus Load in Fecal Samples from the Hong Kong Cohort and Systematic Review and Meta-analysis. Gastroenterology 2020 doi: 10.1053/j.gastro.2020.03.065. [Epub ahead of print].

13.- Ludvigsson JF. Systematic review of COVID-19 in children shows milder cases and a better prognosis than adults. Acta Paediatrica 2020. https://doi.org/10.1111/ apa.15270 [Epub ahead of print].

14.- Khan MA, Khan N, Mustagir G, Rana J, Haque R, Rahman M. COVID-19 infection during pregnancy: a systematic review to summarize possible symptoms, treatments, and pregnancy outcomes. medRxiv 2020:2020.03.31.20049304.

15.- Han C, Duan C, Zhang S, Spiegel B, Shi H, Wang W, et al. Digestive Symptoms in COVID-19 Patients with Mild Disease Severity: Clinical Presentation, Stool Viral RNA Testing, and Outcomes. Am J Gastroenterol 2020 [Preproof].

16.- Chen N, Zhou M, Dong X, Qu J, Gong F, Han Y, et al. Epidemiological and clinical characteristics of 99 cases of 2019 novel coronavirus pneumonia in Wuhan, China: a descriptive study. Lancet 2020;395:507-13.

17. Pan L, Mu M, Yang P, Sun Y, Wang R, Yan J, et al. Clinical Characteristics of COVID-19 Patients With Digestive Symptoms in Hubei, China. Am J Gastroenterol 2020 [Epub ahead of print].

18.- Wang JT, Sheng WH, Fang CT, Chen YC, Wang JL, Yu CJ, et al. Clinical manifestations, laboratory findings, and treatment outcomes of SARS patients. Emerg Infect Dis 2004;10:818-24.

19.- Wan Y, Shang J, Graham R, Baric RS, Li F. Receptor Recognition by the Novel Coronavirus from Wuhan: an Analysis Based on Decade-Long Structural Studies of SARS Coronavirus. J Virol 2020;94 doi: 10.1128/JVI.00127-20 [Epub ahead of print].

20.- Liang W, Feng Z, Rao S, Xiao C, Xue
$X$, Lin Z, et al. Diarrhoea may be underestimated: a missing link in 2019 novel coronavirus. Gut 2020 doi:10.1136/ gutjnl-2020-320832 [Epub ahead of print].

21.- Zhou P, Yang X-L, Wang X-G, Hu B, Zhang L, Zhang W, et al. Discovery of a novel coronavirus associated with the recent pneumonia outbreak in humans and its potential bat origin. Nature 2020;579:270-3.

22.- Letko M, Marzi A, Munster V. Functional assessment of cell entry and receptor usage for SARS-CoV-2 and other lineage $B$ betacoronaviruses. Nat Microbiol 2020;5:562-9.

23.- Hashimoto T, Perlot T, Rehman A, Trichereau J, Ishiguro H, Paolino M, et al. ACE2 links amino acid malnutrition to microbial ecology and intestinal inflammation. Nature 2012;487:477-81.

24.- Xiao F, Tang M, Zheng X, Liu Y, Li X, Shan H. Evidence for Gastrointestinal Infection of SARS-CoV-2.

Gastroenterology 2020 doi: 10.1053/j. gastro.2020.02.055. [Epub ahead of print].

25.- Wang SH, Han P, Xiao F. Manifestations of liver injury in 333 hospitalized patients with coronavirus disease 2019. Chin J Dig, 2020 DOI:10.3760/cma.j.issn. 02541432.2020.03.000 [Preprint].

26.- Zhang C, Shi L, Wang FS. Liver injury in COVID-19: management and challenges. Lancet Gastroenterol Hepatol 2020;5:428-30.

27.- WGO. Gastroenterology Practice in COVID-19 Pandemic, 2020. https://www. worldgastroenterology.org/publications/ewgn/gastroenterology-practice-in-covid19-pandemic

28.- Ling Y, Xu SB, Lin YX, Tian D, Zhu ZQ, Dai FH, et al. Persistence and clearance of viral RNA in 2019 novel coronavirus disease rehabilitation patients. Chin Med $\mathrm{J} 2020$ [Epub ahead of print].

29.- Wu Y, Guo C, Tang L, Hong Z, Zhou J, Dong X, et al. Prolonged presence of SARS-CoV-2 viral RNA in faecal samples. Lancet Gastroenterol Hepatol 2020: 5:434-5.

30.- Wölfel R, Corman VM, Guggemos W, Seilmaier M, Zange S, Müller MA, et al. Virological assessment of hospitalized patients with COVID-2019. Nature 2020 https://doi.org/10.1038/s41586-0202196-x [Epub ahead of print]. 